\title{
A Trusted Framework for Data Security in Cloud Environment
}

\author{
Mannem Sindhuja ${ }^{1}$, Punugoti Pavan Kumar ${ }^{2}$ \\ ${ }^{1,2}$ Computer Science Engineering, Rise Krishna Sai Prakasam Group of Institutions, Ongole, India
}

\begin{abstract}
Data deduplication is one of important data compression techniques for eliminating duplicate copies of repeating data and has been widely used in cloud storage in order to minimize the amount of storage space and save bandwidth. For protection of data security, this paper makes an attempt to primarily address the problem of authorized data deduplication. To protect the confidentiality of important data while supporting deduplication, the convergent encryption technique has been proposed to encrypt the data before outsourcing. Along with the data the privilege level of the user is also checked in order to assure whether he is an authorized user or not. Security analysis demonstrates that our scheme is secure in terms of the definitions specified in the proposed security model. We show that our proposed authorized duplicate check scheme has minimal overhead compared to normal operations. As a proof of concept, we implement a prototype of our proposed authorized duplicate check scheme and conduct tested experiments using our prototype. This paper tries to minimize the data duplication that occurs in hybrid cloud storage by using various techniques.
\end{abstract}

Keywords: Deduplication, authorized duplicate check, confidentiality, hybrid cloud.

\section{Introduction}

To make data management scalable in cloud computing, deduplication has been a well-known technique and has attracted more and more attention recently. Data deduplication is a specialized data compression technique for eliminating duplicate copies of repeating data in storage. The technique is used to improve storage utilization and can also be applied to network data transfers to reduce the number of bytes that must be sent. Instead of keeping multiple data copies with the same content, deduplication eliminates redundant data by keeping only one physical copy and referring other redundant data to that copy. Deduplication can take place at either the file level or the block level. For file level deduplication, it eliminates duplicate copies of the same file. Deduplication can also take place at the block level, which eliminates duplicate blocks of data that occur in non-identical files

Although data deduplication brings a lot of benefits, security and privacy concerns arise as users' sensitive data are susceptible to both insider and outsider attacks .Traditional encryption, while providing data confidentiality, is incompatible with data deduplication. Specifically, traditional encryption requires different users to encrypt their data with their own keys. Thus, identical data copies of different users will lead to different ciphertexts, making deduplication impossible. Convergent encryption has been proposed to enforce data confidentiality while making deduplication feasible. It encrypts/decrypts a data copy with a convergent key, which is obtained by computing the cryptographic hash value of the content of the data copy. After key generation and data encryption, users retain the keys and send the cipher text to the cloud. Since the encryption operation is deterministic and is derived from the data content, identical ldata copies will generate the same convergent key and hence the same cipher text . To prevent unauthorized access, a secure proof of ownership protocol is also needed to provide the proof that the user indeed owns the same file when a duplicate is found. After the proof, subsequent users with the same file will be provided a pointer from the server without needing to upload the same file. A user can download the encrypted file with the pointer from the server, which can only be decrypted by the corresponding data owners with their convergent keys. Thus, convergent encryption allows the cloud to perform deduplication on the ciphertexts and the proof of ownership prevents the unauthorized user to access the file.

\section{Literature Survey}

In archival storage systems, there is a huge amount of duplicate data or redundant data, which occupy significant extra equipments and power consumptions, largely lowering down resources utilization (such as the network bandwidth and storage) and imposing extra burden on management as the scale increases. So data de-duplication, the goal of which is to minimize the duplicate data in the inter level, has been receiving broad attention both in academic and industry in recent years. In this paper, semantic data de-duplication (SDD) is proposed, which makes use of the semantic information in the I/O path (such as file type, file format, application hints and system metadata) of the archival files to direct the dividing a file into semantic chunks (SC). While the main goal of SDD is to maximally reduce the inter file level duplications, directly storing variable SCes into disks will result in a lot of fragments and involve a high percentage of random disk accesses, which is very inefficient. So an efficient data storage scheme is also designed and implemented: SCes are further packaged into fixed sized Objects, which are actually the storage units in the storage devices, so as to speed up the I/O performance as well as ease the data management. Primary experiments have demonstrated that SDD can further reduce the storage space compared with current methods. With the advent of cloud computing, secure data deduplication has attracted much attention recently from research community. Yuan et al. proposed a deduplication system in the cloud storage to reduce the storage size of the tags for integrity check. To enhance the security of deduplication and protect the data confidentiality, Bellare et al. showed how to protect the data confidentiality by transforming the predictable message into 


\section{International Journal of Science and Research (IJSR) \\ ISSN (Online): 2319-7064}

Index Copernicus Value (2013): 6.14 | Impact Factor (2014): 5.611

unpredictable message. In their system, another third party called key server is introduced to generate the file tag for duplicate check. Stanek et al. presented a novel encryption scheme that provides the essential security for popular data and unpopular data. For popular data that are not particularly sensitive, the traditional conventional encryption is performed. Another two-layered encryption scheme with stronger security while supporting deduplication is proposed for unpopular data. In this way, they achieved better trade between the efficiency and security of the out-sourced data. Liet al. addressed the key management issue in block-level deduplication by distributing these keys across multiple servers after encrypting the files.

\section{Overview of the Hybrid Cloud Concepts Hybrid Cloud}

A hybrid cloud is a cloud computing environment in which an organization provides and manages some resources inhouse and has others provided externally .For example, an organization might use a public cloud service, such as Amazon Simple Storage Service(Amazon S3) for archived data but continue to maintain in house storage for operational customer data

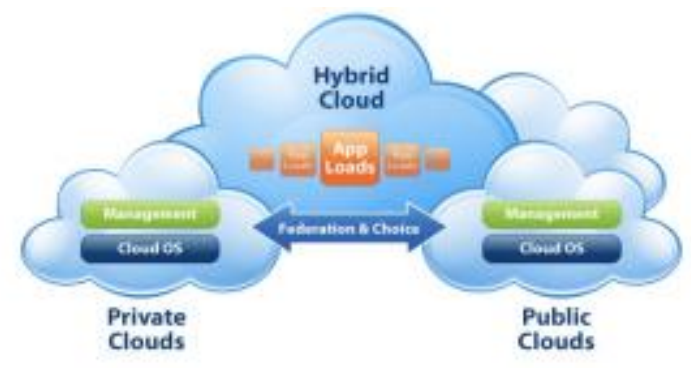

The concept of a hybrid cloud is meant to bridge the gap between high control, high cost "private cloud" and highly callable, flexible, low cost "public cloud".

"Private Cloud" is normally used to describe a VMware deployment in which the hardware and software of the environment is used and managed by a single entity.

The concept of a "Public cloud" usually involves some form of elastic/subscription based resource pools in a hosting provider datacenter that utilizes multi-tenancy. The term public cloud doesn't mean less security, but instead refers to multi-tenancy. The concept revolves heavily around connectivity and data portability. The use cases are numerous: resource burst-ability for seasonal demand, development and testing on a uniform platform without consuming local resources, disaster recovery, and of course excess capacity to make better use of or free up local consumption.

VMware has a key tool for "hybrid cloud" use called "vCloud connector". It is afree plugin that allows the management of public and private clouds within the vSphere client. The tool offers users the ability to manage the console view, power status, and more from a "workloads" tab, and offers the ability to copy virtual machine templates to and from a remote public cloud offering.

\section{Hybrid Cloud for Secure Deduplication}

At a high level, our setting of interest is an enterprise network, consisting of a group of affiliated clients (for example, employees of a company) who will use the S-CSP and store data with deduplication technique. In this setting, deduplication can be frequently used in these settings for data backup and disaster recovery applications while greatly reducing storage space. Such systems are widespread and are often more suitable to user file backup and synchronization applications than richer storage abstractions. There are three entities defined in our system, that is, users, private cloud and S-CSP in public cloud . The S-CSP performs deduplication by checking if the contents of two files are the same and stores only one of them. The access right to a file is defined based on a set of privileges. The exact definition of a privilege varies across applications. For example, we may define a rolebased privilege according to job positions (e.g., Director, Project Lead, and Engineer), or we may define a timebased privilege that specifies a valid time period (e.g., 2014-01- 01 to 2014-01-31) within which a file can be accessed. A user, say Alice, may be assigned two privileges "Director" and "access right valid on 2014- 01-01", so that she can access any file whose access role is "Director" and accessible time period covers 2014- 01-01. Each privilege is represented in the form of a short message called token. Each file is associated with some file tokens, which denote the tag with specified. A user computes and sends duplicate-check tokens to the public cloud for authorized duplicate check. Users have access to the private cloud server, a semitrusted third party which will aid in performing deduplicable encryption by generating file tokens for the requesting users. We will explain further the role of the private cloud server below. Users are also provisioned with per-user encryption keys and credentials

\section{A. Architecture For Authorized Deduplication:}

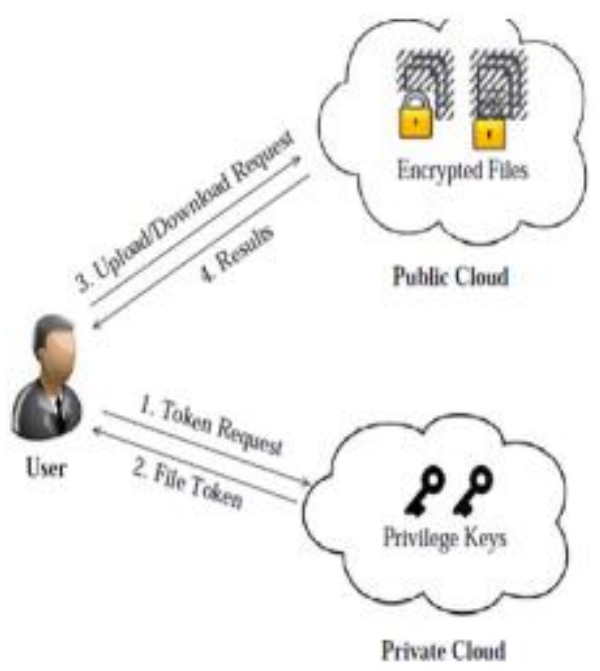

Fig Architecture for Authorized deduplication

In this paper, we will only consider the file level deduplication for simplicity. In another word, we refer a data copy to be a whole file and file-level deduplication which eliminates the storage of any redundant files. Actually, blocklevel deduplication can be easily deduced from filelevel deduplication, Specifically, to upload a file, a user first 


\section{International Journal of Science and Research (IJSR) \\ ISSN (Online): 2319-7064 \\ Index Copernicus Value (2013): 6.14 | Impact Factor (2014): 5.611}

performs the file-level duplicate check. If the file is a duplicate, then all its blocks must be duplicates as well; otherwise, the user further performs the block-level duplicate check and identifies the unique blocks to be uploaded. Each data copy (i.e., a file or a block) is associated with a token for the duplicate check.

- S-CSP. This is an entity that provides a data storage service in public cloud. The S-CSP provides the data outsourcing service and stores data on behalf of the users. To reduce the storage cost, the S-CSP eliminates the storage of redundant data via deduplication and keeps only unique data. In this paper, we assume that S-CSP is always online and has abundant storage capacity and computation power.

- Data Users. A user is an entity that wants to outsource data storage to the S-CSP and access the data later. In a storage system supporting deduplication, the user only uploads unique data but does not upload any duplicate data to save the upload bandwidth, which may be owned by the same user or different users. In the authorized deduplication system, each user is issued a set of privileges in the setup of the system. Each file is protected with the convergent encryption key and privilege keys to realize the authorized deduplication with differential privileges.

- Private Cloud. Compared with the traditional deduplication architecture in cloud computing, this is a new entity introduced for facilitating user's secure usage of cloud service. Specifically, since the computing resources at data user/owner side are restricted and the public cloud is not fully trusted in practice, private cloud is able to provide data user/owner with an execution environment and infrastructure working as an interface between user and the public cloud. The private keys for the privileges are managed by the private cloud, who answers the file token requests from the users. The interface offered by the private cloud allows user to submit files and queries to be securely stored and computed respectively.

Notice that this is a novel architecture for data deduplication in cloud computing, which consists of a twin clouds (i.e., the public cloud and the private cloud). Actually, this hybrid cloud setting has attracted more and more attention recently. For example, an enterprise might use a public cloud service, such as Amazon S3, for archived data, but continue to maintain in-house storage for operational customer data.

Alternatively, the trusted private cloud could be a cluster of virtualized cryptographic co-processors, which are offered as a service by a third party and provide the necessary hardware based security features to implement a remote execution environment trusted by the users.

\section{Conclusion and Future Work}

The notion of authorized data deduplication was proposed to protect the data security by including differential privileges of users in the duplicate check. We also presented several new deduplication constructions supporting authorized duplicate check in hybrid cloud architecture, in which the duplicate check tokens of files are generated by the private cloud serve with private keys. Security analysis demonstrates that our schemes are secure in terms of insider and outsider attacks specified in the proposed security model. As a proof of concept, we implemented a prototype of our proposed authorized duplicate check scheme and conduct testbed experiments on our prototype. We showed that our authorized duplicate check scheme incurs minimal overhead compared to convergent encryption and network transefer.

It excludes the security problems that may arise in the practical deployment of the present model. Also, it increases the national security. It saves the memory by deduplicating the data and thus provides us with sufficient memory. It provides authorization to the private firms and protects the confidentiality of the important data.

\section{References}

[1] P. Anderson and L. Zhang. Fast and secure laptop backups with encrypted de-duplication. In Proc. of USENIX LISA, 2010.

[2] M. Bellare, S. Keelveedhi, and T. Ristenpart.Messagelocked encryption and secure deduplication. In EUROCRYPT, pages 296- 312, 2013.. [3] M. Bellare, S. Keelveedhi, and T. Ristenpart.Dupless: Serveraided encryption for deduplicated storage. In USENIX Security Symposium, 2013.

[3] S. Bugiel, S. Nurnberger, A. Sadeghi, and T. Schneider. Twin clouds: An architecture for secure cloud computing. In Workshop on Cryptography and Security in Clouds (WCSC 2011), 2011.

[4] J. Li, X. Chen, M. Li, J. Li, P. Lee, and W. Lou. Secure deduplication with efficient and reliable convergent key management. In IEEE Transactions on Parallel and Distributed Systems, 2013.

[5] Bugiel, S., N“urnberger, S., Sadeghi, A.-R., Schneider, T.: Twin Clouds: An architecture for secure cloud computing (Extended Abstract). In: Workshop on Cryptography and Security in Clouds (WCSC 2011), March 15-16 (2011)

[6] Chung, K.-M., Kalai, Y., Vadhan, S.: Improved delegation of computation using fully homomorphic encryption. In: Rabin, T. (ed.) CRYPTO 2010. LNCS, vol. 6223, pp. 483-501. Springer, Heidelberg (2010)

[7] Cloud Security Alliance. Top threats to cloud computing, v. 1.0 (2010)

\section{Author Profile}

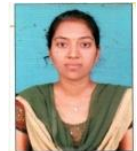

Mannem Sindhuja Obtained the B.Tech. degree in Information Technology (IT) from Buchepalli Venkayamma Subbareddy (BVSR) Engineering College,Chimakurthy. At present persuing the M.Tech in Computer Science and Engineering (CSE) Department at Rise Krishna Sai Prakasam Group Of Institutions, Valluru.

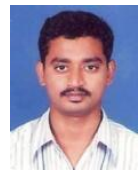

Punugoti. Pavan Kumar obtained the B.Tech Degree in Computer Science and Engineering from Prakasam Engineering College, Kandukur in 2006 and M.Tech from Acharya Najarjuna University in 2010..He has 5 years of Teaching Experience and working in Computer Science and Engineering(CSE) Department at Rise Krishna Sai Gandhi Group Of Institutions, Valluru.

\section{Volume 4 Issue 11, November 2015}

